\title{
Pretreatment with Dual Antiplatelet Therapy in Patients with ST-Elevation Myocardial Infarction
}

Matias B Yudi, MBBS, 1,2 Omar Farouque MBBS, PhD,1,2 Nick Andrianopoulos, MBBS, MBiostat, ${ }^{3}$ Andrew E Ajani, MBBS, MD, ${ }^{2,3}$ Angela Brennan, RN, ${ }^{3}$ Jeffrey Lefkovits, MBBS, ${ }^{3,4}$ Christopher M Reid, BA, MSc, PhD, , 3 William Chan, MBBS, PhD, ${ }^{6}$ Stephen J Duffy, MBBS, PhD, ${ }^{6}$ David J Clark, MBBS, DMedSci, ${ }^{1,2^{*}}$ on behalf of the Melbourne Interventional Group.

$\sqrt{1}$

${ }^{1}$ Department of Cardiology, Austin Health, Melbourne, Australia, ${ }^{2}$ Department of Medicine, University of Melbourne, Melbourne, Australia, ${ }^{3}$ Centre of Cardiovascular Research and Education in Therapeutics (CCRE), Monash University, Melbourne, Australia, ${ }^{4}$ Department of Cardiology, Royal Melbourne Hospital, Melbourne, Australia, ${ }^{5}$ School of Public Health, Curtin University, Perth, Western Australia, ${ }^{6}$ Department of Cardiovascular Medicine, Alfred Health, Melbourne, Australia.

${ }^{*}$ Corresponding author:

Associate Professor David Clark

Director of Interventional Cardiology Research

Austin Health

145 Studley Road

Heidelberg, Victoria 3084

Phone: 0394965527

Fax: $\quad 0394590971$

Email: $\quad$ david.clark@austin.org.au

Keywords: STEMI; antiplatelet therapy; percutaneous coronary intervention.

Word Count: 2,674

This is the author manuscript accepted for publication and has undergone full peer review but has not been through the copyediting, typesetting, pagination and proofreading process, which may lead to differences between this version and the Version record. Please cite this article as doi:10.1002/ ccd.27325. 


\section{ABSTRACT}

Background: The optimal time to administer $\mathrm{P}_{2} \mathrm{Y}_{12}$ inhibitors in patients with ST-elevation myocardial infarction(STEMI) remains to be defined. We sought to assess whether a pretreatment strategy was associated with improved coronary reperfusion and clinical outcomes.

Methods: Consecutive patients from the Melbourne Interventional Group registry (2005-2014) who presented with STEMI and underwent primary PCI were included. Those who received any $\mathrm{P}_{2} \mathrm{Y}_{12}$ inhibitor prior to arrival in the cardiac catheterisation laboratory were included in the pretreatment group. The primary endpoints were the proportion of patients with initial TIMI flow grade $<3$ and in-hospital bleeding. The secondary endpoints were 12-month mortality and major adverse cardiovascular events (MACE).

Results: Of the 2,807 patients included, 892(31.8\%) received pretreatment. Clopidogrel was the most common $\mathrm{P}_{2} \mathrm{Y}_{12}$ inhibitor used (79.6\%). Pretreatment was associated with less thromboaspiration and GPIIb/IIIa inhibitor use (both $\mathrm{p}<0.01)$. Pre-treatment was not associated with lower rates TIMI flow $<3$ on initial angiogram $(78.0 \%$ vs. $80.7 \%, \mathrm{p}=0.18)$ nor with increased in-hospital bleeding (3.6\% vs. 3.9\%,p=0.67). Pretreatment was associated with lower 12month mortality (4.7\% vs. $7.0 \%, \mathrm{p}=0.02)$ but similar MACE rate $(13.0 \%$ vs. $14.1 \%, \mathrm{p}=0.43)$. Multivariate analysis revealed pretreatment was not an independent predictor of 12-month mortality (OR 0.79; 95\% CI 0.5-1.3, p=0.32). Conclusion: Pretreatment with a P2Y 12 inhibitor in patients with STEMI was not routine practice in our Australian cohort and was not associated with improved coronary reperfusion or clinical outcomes. Larger studies are required to definitively ascertain the risk/benefit ratio of dual antiplatelet therapy pretreatment in STEMI. 


\section{BACKGROUND}

Dual antiplatelet therapy is the standard of care for patients with ST-elevation myocardial infarction (STEMI) who are treated with primary percutaneous coronary intervention (PCI).1,2 Although guidelines recommend administration of a $\mathrm{P}_{2} \mathrm{Y}_{12}$ inhibitor, along with aspirin, as soon as possible in STEMI there is still significant clinical equipoise regarding the safety and efficacy of this strategy in the setting of primary PCI.3,4

The more potent $\mathrm{P}_{2} \mathrm{Y}_{12}$ inhibitors, prasugrel and ticagrelor, have shown superiority against clopidogrel in reducing major adverse cardiovascular outcomes at one year. ${ }^{1,2}$ However the exact timing of $\mathrm{P}_{2} \mathrm{Y}_{12}$ inhibitor administration was variable. The recent ATLANTIC Trial aimed but failed to establish the efficacy of prehospital administration of ticagrelor. ${ }^{5}$ Given $10 \%$ of patients with ST-elevation do not have an acute coronary syndrome, and a further $2 \%$ require emergent bypass surgery, there is a clinical rationale for withholding potent antiplatelet therapy until coronary anatomy is known. ${ }^{5}$ Thus, utilizing a well-established and recognized multicenter interventional registry, we aimed to explore the trends and outcomes in patients with STEMI who received pretreatment with dual antiplatelet therapy over a ten-year period.

\section{METHODS}

The study cohort included consecutive patients enrolled in the Melbourne Interventional Group (MIG) registry who underwent primary PCI for management of their index STEMI between January 2005 and December 2014.

The MIG registry is a multicenter PCI registry and has been previously described in detail. 6 Briefly, demographic, clinical, procedural and in-hospital outcome data are prospectively recorded on case-report forms using standardized definitions for all fields with follow up performed at 30 days and 12 months. $^{7}$

The registry is coordinated by the Centre of Cardiovascular Research and Education in Therapeutics; an independent research body within the School of Public Health and Preventive Medicine at Monash University (Melbourne, Australia). An audit of a number of verifiable fields from $5 \%$ of randomly 
selected procedures at each institution is undertaken periodically. ${ }^{8}$ In the most recent audit, 27 fields were assessed with data accuracy of 98\%. This compares favorably to audits from other large registries. ${ }^{9}$ The ethics committee in each participating hospital has approved the MIG registry, including the use of "optout" consent. In this model consent is presumed unless the patient "opts out". All participating sites give each patient a "Patient Information Sheet". If a patient informs a staff member that they do not wish to participate, the patient's data are not collected.

Patients who underwent PCI for STEMI and were treated with aspirin and a $\mathrm{P}_{2} \mathrm{Y}_{12}$ inhibitor were included. Exclusion criteria included: thrombolysis prior to PCI; out-of-hospital cardiac arrest; and use of more than one P2 $\mathrm{Y}_{12}$ inhibitor prior to PCI. STEMI was defined as ECG changes (new ST-segment elevation at the J-point or development of Q-waves in two or more contiguous leads) with confirmed myocardial necrosis (elevation in troponin T or I or CK-MB on at least one occasion within 24 hours from index event).

Acute management of all patients including interventional strategy, stent selection and antithrombotic therapy were left to the discretion of the operator in all procedures.

Patients were divided into two groups based on the timing of $\mathrm{P}_{2} \mathrm{Y}_{12}$ inhibitor (clopidogrel, prasugrel or ticagrelor) administration. Those who received a $\mathrm{P}_{2} \mathrm{Y}_{12}$ inhibitor prior to arrival in the catheterisation laboratory were included in the pre-treatment group. Those who received a $\mathrm{P}_{2} \mathrm{Y}_{12}$ inhibitor during or after the PCI were included in the no pre-treatment group.

The primary efficacy endpoint was the proportion of patients who had a TIMI flow $<3$ in the infarcted artery at initial angiography. The primary safety end-point was in-hospital bleeding.

Secondary outcomes were 12-month mortality and major adverse cardiovascular events (MACE). MACE was defined as the combination of mortality, myocardial infarction (MI), and target vessel revascularization. MI was defined as: an increase in creatine kinase or creatine kinase-MB $\geq 3$ times the upper limit of normal; and/or a significant ST-segment change, development of new $Q$ waves in $\geq 2$ contiguous electrocardiographic leads, or new left branch bundle block pattern in the context of new clinical symptoms. In-hospital 
bleeding was defined as any bleeding requiring a blood transfusion and/or prolonged hospital admission and/or a drop of haemoglobin $>3 \mathrm{~g} / \mathrm{dL}$.

Continuous variables are expressed as mean $\pm \mathrm{SD}$, and categorical data are expressed as numbers/percentages. Continuous variables were compared using Student's t test. Categorical variables were compared using Fisher's exact or Pearson's chi-square tests as appropriate. Variables were tested for linear trends across the years 2005-2014 using Stata's nptrend command. This is a nonparametric test for trend across ordered groups which is an extension of the Wilcoxon rank-sum test. Multivariate logistic regression was used to estimate the adjusted odds ratio and 95\% confidence interval (CI) for 12-month mortality. Univariate variables with $\mathrm{p}<0.10$ were included in multivariate models. The variables used were: pretreatment, age, DTBT, sex, eGFR, hypertension, diabetes, hypercholesterolaemia, family history of coronary disease, previous MI, previous PCI, previous CABG, heart failure, peripheral vascular disease, cerebrovascular disease, left ventricular ejection fraction, multi-vessel CAD, chronic lung disease, cardiogenic shock, glycoprotein IIb/IIIa use, drug-eluting stent use, long stent $(>20 \mathrm{~mm})$, small diameter $(<2.5 \mathrm{~mm})$ and treated lesion location (ostial, bifurcation, left main, LAD, circumflex, right coronary artery, bypass graft). All statistical analyses were performed using Stata 13.1, StataCorp LP, College Station, TX, USA. P-values $<0.05$ were considered to be statistically significant.

\section{RESULTS}

Of the 3,238 consecutive STEMI patients in the MIG registry from 20052014, 2,807 met the inclusion criteria and were included in this study. Pretreatment with a P2Y12 inhibitor, as well as aspirin, occurred in 892 (31.8\%) patients while 1,915 (68.2\%) patients did not receive $\mathrm{P}_{2} \mathrm{Y}_{12}$ inhibitor pretreatment. There was no significant trend in patients receiving pretreatment between 2005 and 2014 despite the availability of prasugrel from 2009 and ticagrelor from 2012 (p-value for trend 0.31). The predominant $\mathrm{P}_{2} \mathrm{Y}_{12}$ inhibitor used was clopidogrel; it was used in $86 \%$ and $77 \%$ of cases in the pretreatment and no pretreatment cohorts. 
Baseline clinical characteristics were similar between the two cohorts (Table 1). However, patients in the pretreatment group were more likely to have a history of previous PCI (13.5\% vs. $10.1 \%$, p<0.01) but had lower rates of Killip class $\geq 2(12.2 \%$ vs. $15.5 \%, \mathrm{p}=0.02)$ and cardiogenic shock (4.5\% vs. $6.7 \%$, $\mathrm{p}=0.02$ ) at presentation. They also had longer median door-to-balloon times (75 vs. 70 minutes, $\mathrm{p}=0.03$ ) and overall ischaemic times (197 vs. 186 minutes, $\mathrm{p}=0.01)$.

Procedural characteristics (Table 2) reveal patients in the pretreatment arm were more likely to undergo transradial PCI (13.8\% vs. $10.4 \%, p=0=0.01)$ but less likely to receive a glycoprotein IIb/IIIa inhibitor (69.6\% vs. 76.2\%, $\mathrm{p}<0.01)$ or be treated with a thrombus aspiration catheter $(18.3 \%$ vs. $24.9 \%$, $\mathrm{p}<0.01)$.

There was no difference in the in-hospital primary endpoint of the proportion of patients with TIMI $<3$ flow at initial angiography (78.0\% vs. 80.7\%, $\mathrm{p}=0.18$; Table 3 ) or the safety endpoint of bleeding (3.6\% vs. 3.9\%, p=0.67). Patients in the pretreatment cohort were less likely to suffer in-hospital complications such as cardiogenic shock, new congestive heart failure and arrhythmias. They also had lower in-hospital mortality $(2.3 \%$ vs. $4.4 \%, p<0.01)$.

Thirty-day and 12-month outcomes are also shown in Table 3. The mortality benefit seen in the pretreatment group during the index admission extended to lower 30-day (2.8\% vs.5.0\%, p<0.01) and 12-month mortality (4.7\% vs. $7.0 \%, p=0.02$ ). There was no difference between the groups in MACE at 30days or 12 -months.

Univariate analysis showed an association between pretreatment and 12month mortality but not for initial TIMI flow, in-hospital bleeding or 12-month MACE. Multivariate logistic regression revealed pretreatment with a $\mathrm{P}_{2} \mathrm{Y}_{12}$ inhibitor was not an independent predictor of 12-month mortality (OR 0.79; 95\% CI 0.49-1.28, p=0.32; Figure 1).

\section{DISCUSSION}

Our study shows there has not been a significant trend for change in pretreatment with dual antiplatelet therapy in STEMI over a 10-year period (2005-2014); the overall pretreatment rate was approximately $32 \%$. An 
association between pretreatment and lower rates of glycoprotein IIb/IIIa inhibitors and thromboaspiration catheter use was observed. $\mathrm{P}_{2} \mathrm{Y}_{12}$ inhibitor pretreatment was safe without a significant increase in major bleeding but was not associated with improved initial TIMI flow on initial angiography. Although, the pretreatment cohort experienced lower short- and medium-term mortality it was not an independent predictor of 12 -month mortality on multivariate analysis, likely due to differences in baseline characteristics between pretreatment versus no pre-treatment groups.

Effective platelet inhibition is essential in the acute management of STEMI with primary PCI.3,4 This concept was validated with the use of glycoprotein $\mathrm{IIb} / \mathrm{III}$ inhibitors where upstream abciximab infusion was associated with improved coronary artery patency on initial angiography, greater success rate of PCI and improved clinical outcomes. ${ }^{10}$ Thus it is intuitive to assume that administration of aspirin and a $\mathrm{P}^{2} \mathrm{Y}_{12}$ inhibitor as early as possible would translate to improved clinical outcomes. Guidelines uniformly recommend pretreatment however, as evident in our study, it is not routine practice. Our $32 \%$ pretreatment rate is consistent with clinical practice in the United States. ${ }^{11}$ Even with availability and increased use of newer, more potent anti-platelet agents, there has not been a trend towards increase pretreatment either in Australia or the United States. ${ }^{11,12}$ This is likely due to the lack of convincing clinical evidence supporting a pretreatment strategy in patients with STEMI. A meta-analysis showed a reduction in major adverse cardiovascular events with clopidogrel pretreatment in patients presenting with STEMI. ${ }^{13}$ In our study, the majority of patients in the pretreatment cohort received clopidogrel. Our pretreatment cohort did experience improved survival in-hospital, at 30 days and at 12 months, however pretreatment was not an independent predictor of lower mortality. This is most likely explained by the lower risk profile of patients in the pretreatment group who had lower rates of cardiogenic shock and Killip class $\geq 2$ at presentation, and experienced lower rates of new heart failure, cardiogenic shock and significant arrhythmias post PCI. Indeed in our multivariate analysis, cardiogenic shock and left ventricular dysfunction were independent predictors of 12 -month mortality. Furthermore, it is plausible 
patients with higher risk features did not receive pretreatment due to perceived higher possibility of requiring urgent bypass surgery.

Our results showed pretreatment with a $\mathrm{P}_{2} \mathrm{Y}_{12}$ inhibitor was not associated with an increase in the proportion of patients with TIMI 3 flow at initial angiography. This is consistent with the results from the ATLANTIC trial where pre-hospital administration of ticagrelor did not translate to increased STsegment resolution or improved initial TIMI flow. ${ }^{5}$ The absence of clinical benefit with pre-hospital use of a more potent $\mathrm{P}_{2} \mathrm{Y}_{12}$ inhibitor raises questions about the overall value of a pretreatment strategy. In the context of STEMI, patients with prasugrel or ticagrelor loading exhibit a delay in the onset of anti-platelet effect with a majority having high on-treatment platelet reactivity at one and two hours post loading dose. ${ }^{14} \mathrm{~A}$ potential solution is administration of crushed ticagrelor or prasugrel tablets. This strategy leads to greater platelet inhibition in STEMI when compared to ingestion of integral tablets. ${ }^{15,16}$ As patients who are undergoing primary PCI do not routinely receive crushed P2 $\mathrm{Y}_{12}$ inhibitors, they are unlikely to have adequate platelet inhibition. This may explain the lack of benefit seen with oral $\mathrm{P}_{2} \mathrm{Y}_{12}$ pretreatment.

As there is an apparent lack of benefit from pretreatment, safety becomes an important issue. In our study there was no difference in major bleeding between the two groups. This would be expected as all patients in our study underwent primary PCI and received dual antiplatelet therapy; the only difference being the timing of the loading dose. The safety concern with pretreatment is not in patients who ultimately undergo PCI but in those who require coronary artery bypass surgery or who have non-coronary causes of STelevation. Although rare, in some of these circumstances dual antiplatelet therapy could be potentially catastrophic. ${ }^{17}$

Contemporary data indicates that $1-1.6 \%$ of STEMI patients require bypass surgery and over $10 \%$ don't require any revascularization. ${ }^{5}$ Thus these patients are unnecessarily placed at increased risk of bleeding. The unacceptable hazard associated with pretreatment prior to assessing suitability for PCI was shown in the ACCOAST trial. Prasugrel pretreatment in patients with non-ST elevation myocardial infarction (NSTEMI) led to significantly higher bleeding rates. ${ }^{18}$ In contrast, ticagrelor pretreatment in the ATLANTIC trial was not 
associated with a bleeding hazard in STEMI, irrespective of the definition used. 5 The difference in population and the larger proportion of patients with STEMI who proceed to PCI may explain the higher risk of bleeding seen with pretreatment in NSTEMI.

We observed that patients in the pretreatment group were less likely to receive a glycoprotein IIb/IIIa inhibitor. It is conceivable the interventionalist may believe that $\mathrm{P}_{2} \mathrm{Y}_{12}$ pretreatment would provide adequate platelet inhibition but this logic is flawed. ${ }^{14}$ Glycoprotein IIb/IIIa inhibitors do provide rapid platelet inhibition but in contemporary practice they were not associated with a reduction in infarct size. ${ }^{19}$ Currently, they are most commonly used in "bail-out" situations such as slow or no-reflow, massive thrombus and/or thrombotic complications. ${ }^{3}$

Cangrelor, a potent intravenous $\mathrm{P}_{2} \mathrm{Y}_{12}$ antagonist with rapid onset and offset, may hold the key in ensuring full platelet inhibition at the time of primary PCI. ${ }^{20}$ Given its rapid onset of action, the infusion can be started once coronary anatomy has been defined thus making pretreatment with oral $\mathrm{P} 2 \mathrm{Y}_{12}$ agents unnecessary. Cangrelor has been shown to decrease the rate of ischaemic events, including stent thrombosis, across a broad spectrum of PCI patients including those with STEMI. ${ }^{20}$ Although mechanistically attractive, the use of cangrelor has not been examined in STEMI against ticagrelor or prasugrel thus it is uncertain whether it would translate to improved clinical outcomes.

There are some limitations to our study that warrant attention. Firstly, we do not record the exact time of $\mathrm{P}_{2} \mathrm{Y}_{12}$ administration, however drug ingestion occurred in-hospital as ambulance initiated dual antiplatelet therapy is not routine care in our setting. Secondly, although there was a significant increase in the use of prasugrel and ticagrelor since these drugs became available in 2010 and 2012 respectively, the majority of our patients over the 10 -year period were treated with clopidogrel. ${ }^{12}$ Furthermore, we did not capture whether the clopidogrel patients received a $300 \mathrm{mg}$ or $600 \mathrm{mg}$ loading dose. Thirdly, we did not include patients with ST-elevation who proceeded to urgent angiography but were not treated with PCI. This limits the generalizability of our findings. Finally, given the observational nature of our study there may be other factors, either unmeasured or unaccounted for, that influenced the outcome. For example, we 
do not measure morphine use although it is known to delay gastric emptying and may be associated with worse outcomes in patients with STEMI receiving ticagrelor pretreatment. ${ }^{5}$

\section{CONCLUSION}

Pretreatment with a $\mathrm{P}_{2} \mathrm{Y}_{12}$ inhibitor in patients with STEMI is not routine practice in our Australian cohort. Moreover, this strategy is not associated with improved initial TIMI flow at time of angiography or clinical outcomes when baseline clinical and procedural variables are taken into account. Our findings do not provide adequate justification for a strategy of pretreatment. Larger studies are required to definitively ascertain the risk/benefit ratio of dual antiplatelet therapy pretreatment in STEMI
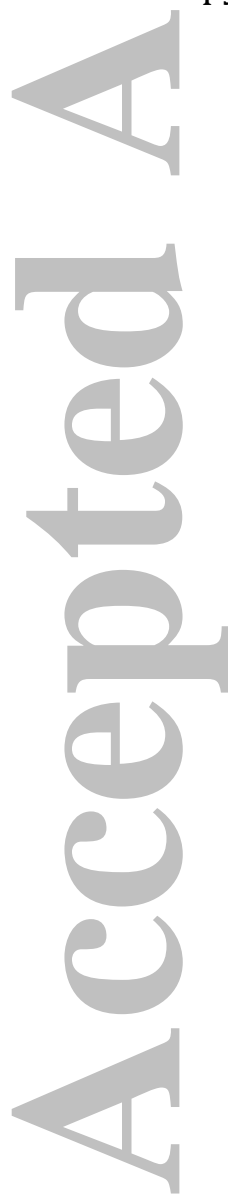
Figure 1. Multivariate Logistic Regression for 12-Month Mortality
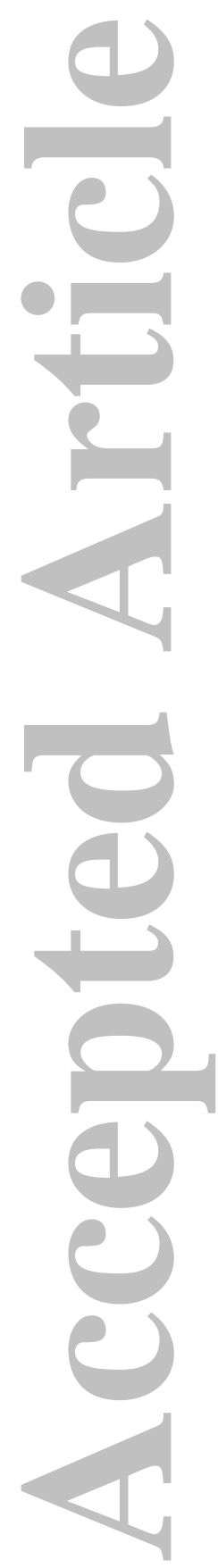


\section{Melbourne Interventional Group Investigators}

MIG Data Management Centre, CCRE, Monash University:

Professor Chris Reid, Dr Nick Andrianopoulos, Ms Angela Brennan, Ms Vibhasha Chand, Dr Diem Dinh.

\section{MIG Steering Committee:}

Professor Chris Reid, A/Professor Andrew Ajani, Dr Stephen Duffy, Dr David Clark, Professor Gishel New, Dr Melanie Freeman, Dr Chin Hiew, Dr Nick Andrianopoulos, Dr Ernesto Oqueli, Ms Angela Brennan.

The following Investigators, Data Managers and Institutions participated in the MIG Database:

Alfred Hospital: SJ Duffy, JA Shaw, A Walton, A Dart, A Broughton, J Federman, C Keighley, C Hengel, KH Peter, D Stub, W Chan, J O’Brien, L Selkrig, K Rankin, R Vandernet, R Huntington, S Pally;

Austin Hospital: DJ Clark, O Farouque, M Horrigan, J Johns, L Oliver, J Brennan, R Chan, G Proimos, T Dortimer, B Chan, V Nadurata, R Huq, D Fernando, A Al-Fiadh, M Yudi, H Sugumar, J Ramchand, H Han. S Picardo, L Brown;

Ballarat Base Hospital: E Oqueli, C Hengel, A Sharma, B Zhu, N Ryan, T Harrison;

Box Hill Hospital: G New, L Roberts, M Freeman, M Rowe, G Proimos, Y Cheong, C Goods, D Fernando, A Teh, S Parfrey, J Ramzy, A Koshy, P Venkataraman, D Flannery;

Geelong Hospital: C Hiew, M Sebastian, T Yip, Michael Mok, C Jaworski, A Hutchinson, C Cimenkaya, P Ngu, B Khialani, H Salehi, M Turner, J Dyson, B McDonald, D Van Den Nouwelant, K Halliburton;

Monash University: H Krum, C Reid, N Andrianopoulos, AL Brennan, V Chand, D Dinh, BP Yan;

Royal Melbourne Hospital: AE Ajani, R Warren, D Eccleston, J Lefkovits, R Iyer, R Gurvitch, W Wilson, M Brooks, S Biswas, J Yeoh.

\section{Acknowledgements:}

The Melbourne Interventional Group acknowledges unrestricted educational grant funding from: Abbott Vascular, Astra-Zeneca, Medtronic, MSD, Pfizer, Servier, and The Medicines Company. These companies do not have access to the data, and do not have the right to review manuscripts before publication.

Dr. Yudi is supported by a combined National Health and Medical Research Council Postgraduate Scholarship (APP 1115163) and a National Heart Foundation Health Professional Scholarship (Award ID 101130). Dr. Duffy's \& Professor Reid's work is funded by National Health and Medical Research Council of Australia Grants.

The Melbourne Interventional Group acknowledges and thanks all patients who have been involved in the registry. 
Conflicts of Interests: The authors report no relationships that could be construed as a conflict of interest.

\section{REFERENCES}

1. Wiviott SD, Braunwald E, McCabe CH, Montalescot G, Ruzyllo W, Gottlieb S, Neumann FJ, Ardissino D, De Servi S, Murphy SA, Riesmeyer J, Weerakkody G, Gibson CM, Antman EM, Investigators T-T. Prasugrel versus clopidogrel in patients with acute coronary syndromes. The New England journal of medicine 2007;357:2001-2015.

2. Wallentin L, Becker RC, Budaj A, Cannon CP, Emanuelsson H, Held C, Horrow J, Husted S, James S, Katus H, Mahaffey KW, Scirica BM, Skene A, Steg PG, Storey RF, Harrington RA, Investigators P, Freij A, Thorsen M. Ticagrelor versus clopidogrel in patients with acute coronary syndromes. The New England journal of medicine 2009;361:1045-1057.

3. Steg PG, James SK, Atar D, Badano LP, Blomstrom-Lundqvist C, Borger MA, Di Mario C, Dickstein K, Ducrocq G, Fernandez-Aviles F, Gershlick AH, Giannuzzi P, Halvorsen S, Huber K, Juni P, Kastrati A, Knuuti J, Lenzen MJ, Mahaffey KW, Valgimigli M, van 't Hof A, Widimsky P, Zahger D. ESC Guidelines for the management of acute myocardial infarction in patients presenting with STsegment elevation. European heart journal 2012;33:2569-2619.

4. O'Gara PT, Kushner FG, Ascheim DD, Casey DE, Jr., Chung MK, de Lemos JA, Ettinger SM, Fang JC, Fesmire FM, Franklin BA, Granger CB, Krumholz HM, Linderbaum JA, Morrow DA, Newby LK, Ornato JP, Ou N, Radford MJ, TamisHolland JE, Tommaso JE, Tracy CM, Woo YJ, Zhao DX, Force CAT. 2013 ACCF/AHA guideline for the management of ST-elevation myocardial infarction: executive summary: a report of the American College of Cardiology Foundation/American Heart Association Task Force on Practice Guidelines. Circulation 2013;127:529-555.

5. Montalescot G, van 't Hof AW, Lapostolle F, Silvain J, Lassen JF, Bolognese L, Cantor WJ, Cequier A, Chettibi M, Goodman SG, Hammett CJ, Huber K, Janzon M, Merkely B, Storey RF, Zeymer U, Stibbe O, Ecollan P, Heutz WM, Swahn E, Collet JP, Willems FF, Baradat C, Licour M, Tsatsaris A, Vicaut E, Hamm CW, Investigators A. Prehospital ticagrelor in ST-segment elevation myocardial infarction. The New England journal of medicine 2014;371:1016-1027.

6. Ajani AE, Szto G, Duffy SJ, Eccleston D, Clark DJ, Lefkovits J, Chew DP, Warren R, Black A, New G, Walton A, Lew R, Shaw J, Horrigan M, Sebastian M, Yan BP, Brennan A, Meehan A, Reid C, Krum H, Melbourne Interventional Group i. The foundation and launch of the Melbourne Interventional Group: a collaborative interventional cardiology project. Heart, lung \& circulation 2006;15:44-47. 7. Chan W, Clark DJ, Ajani AE, Yap CH, Andrianopoulos N, Brennan AL, Dinh DT, Shardey GC, Smith JA, Reid CM, Duffy SJ. Progress towards a National Cardiac Procedure Database--development of the Australasian Society of Cardiac and Thoracic Surgeons (ASCTS) and Melbourne Interventional Group (MIG) registries. Heart, lung \& circulation 2011;20:10-18.

8. Andrianopoulos N, Dinh D, Duffy SJ, Clark DJ, Brennan AL, Chan W, Shardey GC, Smith JA, Yap CH, Buxton BF, Ajani AE, Reid CM. Quality control activities associated with registries in interventional cardiology and surgery. Heart, lung \& circulation 2011;20:180-186. 
9. Lagerqvist B, James SK, Stenestrand U, Lindback J, Nilsson T, Wallentin L, Group SS. Long-term outcomes with drug-eluting stents versus bare-metal stents in Sweden. The New England journal of medicine 2007;356:1009-1019.

10. Montalescot G, Barragan P, Wittenberg O, Ecollan P, Elhadad S, Villain P, Boulenc JM, Morice MC, Maillard L, Pansieri M, Choussat R, Pinton P, Angioplasty AIAbD, Stenting in Myocardial Infarction Regarding A, Long-Term F-u. Platelet glycoprotein IIb/IIIa inhibition with coronary stenting for acute myocardial infarction. The New England journal of medicine 2001;344:1895-1903.

11. Fan $W$, Plent $S$, Prats J, Deliargyris EN. Trends in P2Y12 Inhibitor Use in Patients Referred for Invasive Evaluation of Coronary Artery Disease in Contemporary US Practice. The American journal of cardiology 2016;117:14391443.

12. Yudi MB, Clark DJ, Farouque O, Eccleston D, Andrianopoulos N, Duffy SJ, Brennan A, Lefkovits J, Ramchand J, Yip T, Oqueli E, Reid CM, Ajani AE, Melbourne Interventional G. Clopidogrel, prasugrel or ticagrelor in patients with acute coronary syndromes undergoing percutaneous coronary intervention. Internal medicine journal 2016;46:559-565.

13. Bellemain-Appaix A, O'Connor SA, Silvain J, Cucherat M, Beygui F, Barthelemy O, Collet JP, Jacq L, Bernasconi F, Montalescot G, Group A. Association of clopidogrel pretreatment with mortality, cardiovascular events, and major bleeding among patients undergoing percutaneous coronary intervention: a systematic review and meta-analysis. Jama 2012;308:2507-2516.

14. Alexopoulos D, Xanthopoulou I, Gkizas V, Kassimis G, Theodoropoulos KC, Makris G, Koutsogiannis N, Damelou A, Tsigkas G, Davlouros P, Hahalis G. Randomized assessment of ticagrelor versus prasugrel antiplatelet effects in patients with ST-segment-elevation myocardial infarction. Circulation Cardiovascular interventions 2012;5:797-804.

15. Parodi G, Xanthopoulou I, Bellandi B, Gkizas V, Valenti R, Karanikas S, Migliorini A, Angelidis C, Abbate R, Patsilinakos S, Baldereschi GJ, Marcucci R, Gensini GF, Antoniucci D, Alexopoulos D. Ticagrelor crushed tablets administration in STEMI patients: the MOJITO study. Journal of the American College of Cardiology 2015;65:511-512.

16. Rollini F, Franchi F, Hu J, Kureti M, Aggarwal N, Durairaj A, Park Y, Seawell M, Cox-Alomar P, Zenni MM, Guzman LA, Suryadevara S, Antoun P, Bass TA, Angiolillo DJ. Crushed Prasugrel Tablets in Patients With STEMI Undergoing Primary Percutaneous Coronary Intervention: The CRUSH Study. Journal of the American College of Cardiology 2016;67:1994-2004.

17. Chatterjee S. ECG Changes in Subarachnoid Haemorrhage: A Synopsis. Neth Heart J 2011;19:31-34.

18. Montalescot $G$, Bolognese $L$, Dudek $D$, Goldstein $P$, Hamm $C$, Tanguay JF, ten Berg JM, Miller DL, Costigan TM, Goedicke J, Silvain J, Angioli P, Legutko J, Niethammer M, Motovska Z, Jakubowski JA, Cayla G, Visconti LO, Vicaut E, Widimsky P, Investigators A. Pretreatment with prasugrel in non-ST-segment elevation acute coronary syndromes. The New England journal of medicine 2013;369:999-1010.

19. Mehilli J, Kastrati A, Schulz S, Frungel S, Nekolla SG, Moshage W, Dotzer F, Huber K, Pache J, Dirschinger J, Seyfarth M, Martinoff S, Schwaiger M, Schomig A, Bavarian Reperfusion Alternatives Evaluation-3 Study I. Abciximab in patients with acute ST-segment-elevation myocardial infarction undergoing primary 
percutaneous coronary intervention after clopidogrel loading: a randomized double-blind trial. Circulation 2009;119:1933-1940.

20. Bhatt DL, Stone GW, Mahaffey KW, Gibson CM, Steg PG, Hamm CW, Price MJ, Leonardi S, Gallup D, Bramucci E, Radke PW, Widimsky P, Tousek F, Tauth J, Spriggs D, McLaurin BT, Angiolillo DJ, Genereux P, Liu T, Prats J, Todd M, Skerjanec S, White HD, Harrington RA, Investigators CP. Effect of platelet inhibition with cangrelor during PCI on ischemic events. The New England journal of medicine 2013;368:1303-1313.
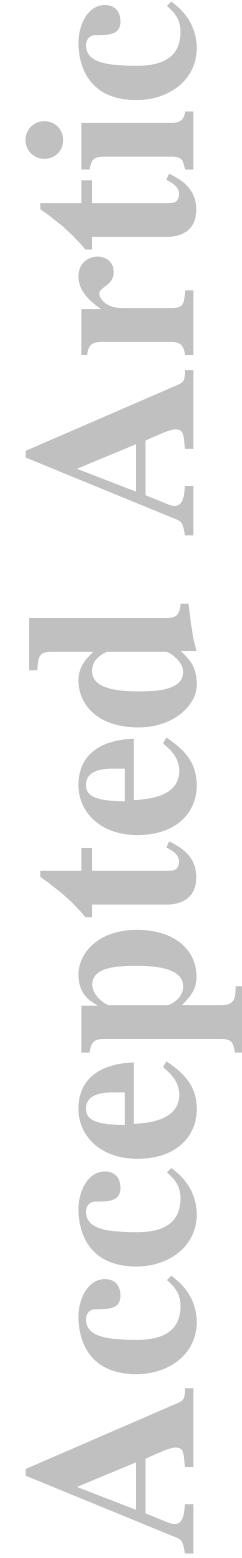
TABLE 1. Baseline Clinical and Procedural Characteristics

\begin{tabular}{|c|c|c|c|}
\hline Characteristics & $\begin{array}{l}\text { Pretreatment } \\
\qquad \mathrm{N}=892\end{array}$ & $\begin{array}{c}\text { No Pretreatment } \\
N=1,915\end{array}$ & p-value \\
\hline Age (mean years $\pm S D$ ) & $63.6 \pm 12.4$ & $63.9 \pm 13.0$ & 0.69 \\
\hline Age $>75$ years & $187(21.0)$ & $455(23.8)$ & 0.10 \\
\hline Male & $688(77.1)$ & $1462(76.3)$ & 0.65 \\
\hline Body mass index & $27.6 \pm 4.9$ & $27.3 \pm 4.8$ & 0.36 \\
\hline Current smoker & $328(37.1)$ & $650(34.4)$ & 0.17 \\
\hline Hypertension & $467(52.4)$ & 989 (51.7) & 0.76 \\
\hline Hypercholesterolaemia & $428(48.0)$ & $965(50.5)$ & 0.22 \\
\hline Diabetes mellitus & $145(16.3)$ & $274(14.3)$ & 0.18 \\
\hline Family history of CAD & $331(38.1)$ & $651(34.7)$ & 0.08 \\
\hline Previous MI & $128(14.4)$ & $242(12.6)$ & 0.21 \\
\hline Previous $\mathrm{PCl}$ & $120(13.5)$ & $194(10.1)$ & $<0.01$ \\
\hline Previous bypass surgery & $21(2.4)$ & $35(1.8)$ & 0.35 \\
\hline Heart Failure & $10(1.1)$ & $24(1.3)$ & 0.76 \\
\hline Peripheral vascular disease & $31(3.5)$ & $54(2.8)$ & 0.35 \\
\hline Stroke & $32(3.6)$ & $90(4.7)$ & 0.18 \\
\hline Chronic Lung Disease & $50(5.6)$ & $159(8.3)$ & 0.01 \\
\hline eGFR $\geq 60 \mathrm{ml} / \mathrm{min} / 1.73 \mathrm{~m}^{2}$ & $696(80.3)$ & $1408(76.7)$ & \\
\hline eGFR $30-60 \mathrm{ml} / \mathrm{min} / 1.73 \mathrm{~m}^{2}$ & $154(17.8)$ & $387(21.1)$ & \\
\hline eGFR $<30 \mathrm{ml} / \mathrm{min} / 1.73 \mathrm{~m}^{2}$ & $17(2.0)$ & $41(2.2)$ & 0.11 \\
\hline $\begin{array}{l}\text { New heart failure } \\
(<2 \text { weeks) }\end{array}$ & $28(3.1)$ & $89(4.7)$ & 0.06 \\
\hline Killip Class $\geq 2$ & $106(12.2)$ & $290(15.5)$ & 0.02 \\
\hline Cardiogenic Shock & $40(4.5)$ & $129(6.7)$ & 0.02 \\
\hline $\begin{array}{l}\text { Symptom-to-balloon time } \\
\text { (minutes; median, IQR) }\end{array}$ & $197(149,267)$ & $186(142,261)$ & 0.01 \\
\hline $\begin{array}{l}\text { Door-to-balloon time } \\
\text { (minutes; median, IQR) }\end{array}$ & $75(50,102)$ & $70(45,98)$ & 0.03 \\
\hline DTBT $<90$ minutes & $577(65.5)$ & 1198 (67.0) & 0.44 \\
\hline Pre-hospital MI activation & $103(43.1)$ & $307(44.7)$ & 0.67 \\
\hline
\end{tabular}

Expressed as count (percentages) unless otherwise stated. CAD = coronary artery disease. $\mathrm{MI}=$ myocardial infarction. $\mathrm{PCl}=$ percutaneous coronary intervention. eGFR = estimated glomerular filtration rate. DTBT = door-to-balloon-time. 
TABLE 2. Procedural Characteristics

\begin{tabular}{|c|c|c|c|}
\hline Characteristics & $\begin{array}{l}\text { Pretreatment } \\
\qquad \mathrm{N}=892\end{array}$ & $\begin{array}{c}\text { No Pretreatment } \\
N=1,915\end{array}$ & p-value \\
\hline Radial Approach & $123(13.8)$ & $200(10.4)$ & 0.01 \\
\hline Femoral Approach & 766 (85.9) & $1713(89.5)$ & 0.01 \\
\hline $\begin{array}{l}\text { Glycoprotein IIb/IIla } \\
\text { Inhibitor use }\end{array}$ & $621(69.6)$ & $1458(76.2)$ & $<0.01$ \\
\hline Periprocedural Heparin & $886(99.3)$ & 1893 (98.9) & 0.24 \\
\hline Aspirin & $892(100)$ & $1914(100)$ & - \\
\hline $\begin{array}{l}\text { Ejection Fraction } \\
\text { (mean\% } \% \text { SD) }\end{array}$ & $52.0 \pm 12.1$ & $50.6 \pm 11.2$ & 0.06 \\
\hline Ejection Fraction $>45 \%$ & $564(67.2)$ & $1138(64.0)$ & 0.12 \\
\hline Multivessel CAD & $488(54.7)$ & $977(51.2)$ & 0.08 \\
\hline Left main disease & $20(7.3)$ & $44(8.0)$ & 0.73 \\
\hline \multicolumn{4}{|l|}{ Culprit vessel PCI } \\
\hline LAD artery & $373(36.2)$ & $809(36.6)$ & 0.82 \\
\hline Circumflex artery & $88(8.5)$ & $211(9.6)$ & 0.35 \\
\hline Right coronary artery & $424(41.2)$ & $897(40.6)$ & 0.77 \\
\hline Left Main coronary artery & $3(0.3)$ & $16(0.7)$ & 0.13 \\
\hline Graft & $13(1.3)$ & $18(0.8)$ & 0.22 \\
\hline $\begin{array}{l}\text { ACC/AHA Lesion } \\
\text { Classification B2/C }\end{array}$ & $733(71.2)$ & $1503(68.1)$ & 0.08 \\
\hline Estimated lesion length & $20.6 \pm 8.7$ & $20.3 \pm 9.1$ & 0.15 \\
\hline Bare Metal Stent & 481 (53.9) & $1026(53.6)$ & \\
\hline Drug Eluting Stent & 356 (39.9) & $759(39.6)$ & \\
\hline Angioplasty alone & $55(6.2)$ & $130(6.8)$ & 0.83 \\
\hline Number of stents inserted & $1.2 \pm 0.6$ & $1.2 \pm 0.6$ & 0.89 \\
\hline Thromboaspiration Use & $156(18.3)$ & $428(24.9)$ & $<0.01$ \\
\hline Successful lesion PCI & $987(96.1)$ & $2006(96.6)$ & 0.5 \\
\hline
\end{tabular}

Expressed as count (percentages) unless otherwise stated. CAD = coronary artery disease. $\mathrm{PCl}=$ percutaneous coronary intervention. $\mathrm{LAD}=$ left anterior descending. 
TABLE 3. Acute and 1-year Outcomes

\begin{tabular}{|c|c|c|c|}
\hline Characteristics & $\begin{array}{l}\text { Pretreatment } \\
\qquad \mathrm{N}=892\end{array}$ & $\begin{array}{c}\text { No Pretreatment } \\
N=1,915\end{array}$ & p-value \\
\hline Pre-PCI TIMI flow <3 & $665(78.0)$ & $1491(80.7)$ & 0.18 \\
\hline Successful lesion PCl & $987(96.1)$ & 2006 (96.6) & 0.5 \\
\hline Post-PCI TIMI flow & & & 0.464 \\
\hline 0 & $28(2.7)$ & 42 (1.9) & \\
\hline 1 & $9(0.9)$ & $19(0.9)$ & \\
\hline 2 & $35(3.4)$ & $85(3.9)$ & \\
\hline 3 & $958(93.0)$ & 2061(93.4) & \\
\hline Acute Closure & $11(1.1)$ & $16(0.7)$ & 0.32 \\
\hline Dissection & $42(4.1)$ & $82(3.7)$ & 0.62 \\
\hline Perforation & $5(0.5)$ & $6(0.3)$ & 0.33 \\
\hline No-reflow & $58(5.7)$ & $104(5.0)$ & 0.05 \\
\hline - Transient no-reflow & $38(3.7)$ & $85(4.1)$ & \\
\hline - Persistent no-reflow & $20(1.9)$ & $19(0.9)$ & \\
\hline Emergency $\mathrm{PCl}$ & $10(1.1)$ & $17(0.9)$ & 0.6 \\
\hline Stent Thrombosis & $7(0.9)$ & $12(0.8)$ & 0.73 \\
\hline Unplanned CABG & $13(1.5)$ & $29(1.5)$ & 0.9 \\
\hline Cardiogenic shock & $32(3.6)$ & $122(6.4)$ & $<0.01$ \\
\hline Arrhythmia & $117(13.1)$ & $358(18.7)$ & $<0.01$ \\
\hline Stroke & $6(0.7)$ & $10(0.5)$ & 0.62 \\
\hline New Renal impairment & $10(2.1)$ & $36(2.1)$ & 0.13 \\
\hline $\begin{array}{l}\text { New Congestive Heart } \\
\text { Failure }\end{array}$ & $46(5.2)$ & $151(7.9)$ & $<0.01$ \\
\hline Length of stay (days) & $4.9 \pm 4.4$ & $4.8 \pm 4.3$ & 0.71 \\
\hline In-hospital bleeding & $32(3.6)$ & 75 (3.9) & 0.67 \\
\hline In-hospital Mortality & $21(2.3)$ & $85(4.4)$ & 0.007 \\
\hline In-hospital MACE & $45(5.0)$ & $125(6.5)$ & 0.13 \\
\hline 12-month Mortality & $42(4.7)$ & $133(7.0)$ & 0.02 \\
\hline 12-month MI & $29(3.3)$ & 75 (3.9) & 0.39 \\
\hline 12M TVR & $66(7.4)$ & $124(6.5)$ & 0.37 \\
\hline 12M MACE & $116(13.0)$ & $270(14.1)$ & 0.43 \\
\hline 30D Mortality & $25(2.8)$ & $96(5.0)$ & 0.007 \\
\hline 30D MI & $13(1.5)$ & $36(1.9)$ & 0.43 \\
\hline 30D TVR & $34(3.8)$ & $62(3.2)$ & 0.44 \\
\hline 30D MACE & $62(7.0)$ & $161(8.4)$ & 0.18 \\
\hline
\end{tabular}

Expressed as count (percentages) unless otherwise stated. $\mathrm{PCl}=$ percutaneous coronary intervention. $\mathrm{TIMI}=$ thrombolysis in myocardial infarction. $\mathrm{CABG}=$ coronary artery bypass surgery. $\mathrm{MACE}=$ major adverse cardiovascular events. $\mathrm{MI}=$ myocardial infarction. TVR = target vessel revascularization. 


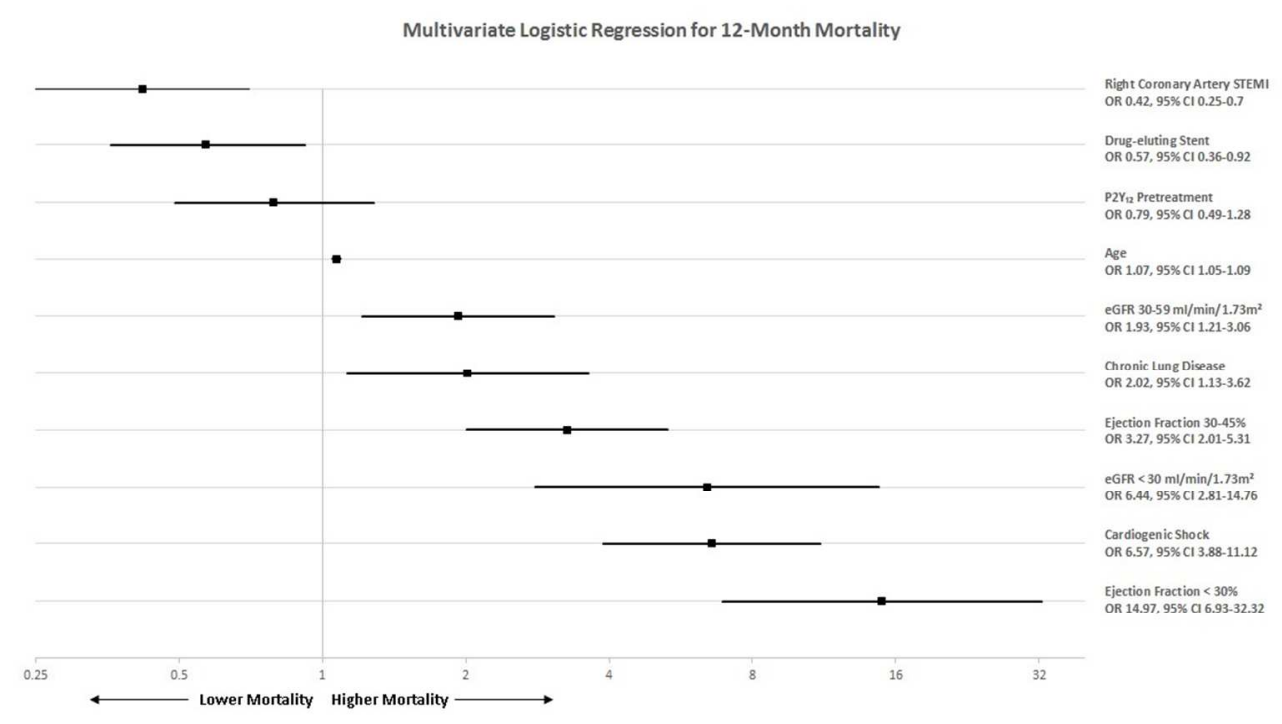

Multivariate Logistic Regression for 12-Month Mortality $300 \times 168 \mathrm{~mm}(96 \times 96 \mathrm{DPI})$

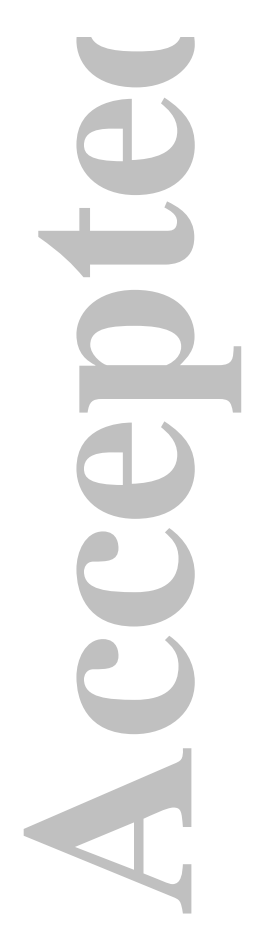




\section{Pretreatment with Dual Antiplatelet Therapy in Patients with ST-Elevation Myocardial Infarction}

Matias B Yudi, MBBS, 1,2 Omar Farouque MBBS, PhD,1,2 Nick Andrianopoulos, MBBS, MBiostat, ${ }^{3}$ Andrew E Ajani, MBBS, MD, ${ }^{2,3}$ Angela Brennan, RN, ${ }^{3}$ Jeffrey Lefkovits, MBBS, ${ }^{3,4}$ Christopher M Reid, BA, MSc, PhD, , 3 William Chan, MBBS, PhD, ${ }^{6}$ Stephen J Duffy, MBBS, PhD, ${ }^{6}$ David J Clark, MBBS, DMedSci, ${ }^{1,2^{*}}$ on behalf of the Melbourne Interventional Group.

$(1$

${ }^{1}$ Department of Cardiology, Austin Health, Melbourne, Australia, ${ }^{2}$ Department of Medicine, University of Melbourne, Melbourne, Australia, ${ }^{3}$ Centre of Cardiovascular Research and Education in Therapeutics (CCRE), Monash University, Melbourne, Australia, ${ }^{4}$ Department of Cardiology, Royal Melbourne Hospital, Melbourne, Australia, ${ }^{5}$ School of Public Health, Curtin University, Perth, Western Australia, ${ }^{6}$ Department of Cardiovascular Medicine, Alfred Health,

Melbourne, Australia.

${ }^{*}$ Corresponding author:

Associate Professor David Clark

Director of Interventional Cardiology Research

Austin Health

145 Studley Road

Heidelberg, Victoria 3084

Phone: 0394965527

Fax: $\quad 0394590971$

Email: david.clark@austin.org.au

Keywords: STEMI; antiplatelet therapy; percutaneous coronary intervention.

Word Count: 2,674 


\section{ABSTRACT}

Background: The optimal time to administer $\mathrm{P}_{2} \mathrm{Y}_{12}$ inhibitors in patients with ST-elevation myocardial infarction(STEMI) remains to be defined. We sought to assess whether a pretreatment strategy was associated with improved coronary reperfusion and clinical outcomes.

Methods: Consecutive patients from the Melbourne Interventional Group registry (2005-2014) who presented with STEMI and underwent primary PCI were included. Those who received any $\mathrm{P}_{2} \mathrm{Y}_{12}$ inhibitor prior to arrival in the cardiac catheterisation laboratory were included in the pretreatment group. The primary endpoints were the proportion of patients with initial TIMI flow grade $<3$ and in-hospital bleeding. The secondary endpoints were 12-month mortality and major adverse cardiovascular events (MACE).

Results: Of the 2,807 patients included, 892(31.8\%) received pretreatment. Clopidogrel was the most common P2Y12 inhibitor used (79.6\%). Pretreatment was associated with less thromboaspiration and GPIIb/IIIa inhibitor use (both $\mathrm{p}<0.01$ ). Pre-treatment was not associated with lower rates TIMI flow $<3$ on initial angiogram $(78.0 \%$ vs. 80.7\%,p=0.18) nor with increased in-hospital bleeding (3.6\% vs. 3.9\%,p=0.67). Pretreatment was associated with lower 12month mortality (4.7\% vs. $7.0 \%, \mathrm{p}=0.02)$ but similar MACE rate $(13.0 \%$ vs. $14.1 \%, \mathrm{p}=0.43)$. Multivariate analysis revealed pretreatment was not an independent predictor of 12-month mortality (OR 0.79; 95\% CI 0.5-1.3, p=0.32). Conclusion: Pretreatment with a $\mathrm{P}_{2} \mathrm{Y}_{12}$ inhibitor in patients with STEMI was not routine practice in our Australian cohort and was not associated with improved coronary reperfusion or clinical outcomes.-Larger studies are required to definitively ascertain the risk/benefit ratio of dual antiplatelet therapy pretreatment in STEMI. 
Therefore, determining the extent of coronary artery disease prior to $\mathrm{P} 2 \mathrm{Y}_{12}$ inhibitor administration would avoid exposure of potent antiplatelet agents in patients who require urgent bypass surgery or have non-coronary causes of STelevation.
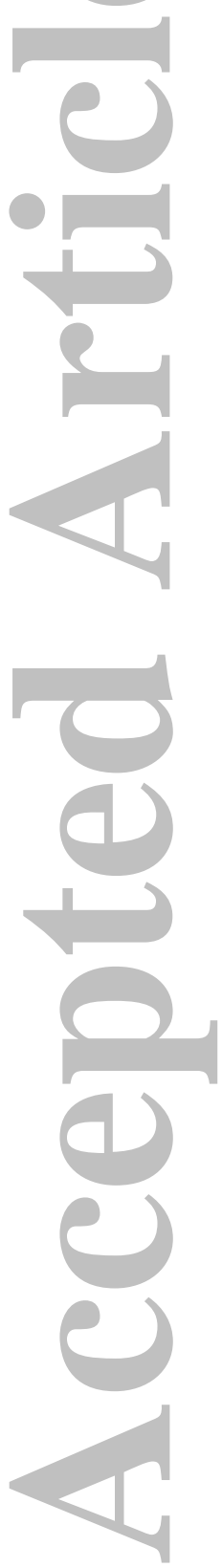


\section{BACKGROUND}

Dual antiplatelet therapy is the standard of care for patients with ST-elevation myocardial infarction (STEMI) who are treated with primary percutaneous coronary intervention (PCI). ${ }^{1,2}$ Although guidelines recommend administration of a $\mathrm{P}_{2} \mathrm{Y}_{12}$ inhibitor, along with aspirin, as soon as possible in STEMI there is still significant clinical equipoise regarding the safety and efficacy of this strategy in the setting of primary PCI.3,4

The more potent $\mathrm{P}_{2} \mathrm{Y}_{12}$ inhibitors, prasugrel and ticagrelor, have shown superiority against clopidogrel in reducing major adverse cardiovascular outcomes at one year. ${ }^{1,2}$ The However the exact timing of $\mathrm{P}_{2} \mathrm{Y}_{12}$ inhibitor administration was variable. in relation to timing of primary PCI in these megatrials. In the PLATelet inhibition and patient Outcomes (PLATO) trial, patients received a $\mathrm{P}_{2} \mathrm{Y}_{12}$ inhibitor at time of diagnosis while in TRITON-TIMI 38, a P2Y 12 inhibitor could be given in STEMI from diagnosis up to one-hour post PCI. ${ }^{1,2}$-The recent ATLANTIC Trial aimed but failed to establish the efficacy of prehospital administration of ticagrelor. Although it was safe, without any significant increase in bleeding, it did not meet its primary endpoint of greater ST-segment resolution nor improved Thrombolysis in Myocardial Infarction (TIMI) flow at initial angiography. ${ }^{5}$ Given $10 \%$ of patients with ST-elevation do not have an acute coronary syndrome, and a further $2 \%$ require emergent bypass surgery, there is a clinical rationale for withholding potent antiplatelet therapy until coronary anatomy is known. ${ }^{5}$ Thus, utilizing a well-established and recognized multicenter interventional registry, we aimed to explore the trends and outcomes in patients with STEMI who received pretreatment with dual antiplatelet therapy over a ten-year period.

\section{METHODS}

The study cohort included consecutive patients enrolled in the Melbourne Interventional Group (MIG) registry who underwent primary PCI for management of their index STEMI between January 2005 and December 2014.

The MIG registry is a multicenter PCI registry and has been previously described in detail. 6 Briefly, demographic, clinical, procedural and in-hospital 
outcome data are prospectively recorded on case-report forms using standardized definitions for all fields with follow up performed at 30 days and 12 months. $^{7}$

The registry is coordinated by the Centre of Cardiovascular Research and Education in Therapeutics; an independent research body within the School of Public Health and Preventive Medicine at Monash University (Melbourne, Australia). An audit of a number of verifiable fields from $5 \%$ of randomly selected procedures at each institution is undertaken periodically. ${ }^{8}$ In the most recent audit, 27 fields were assessed with data accuracy of 98\%. This compares favorably to audits from other large registries. ${ }^{9}$ The ethics committee in each participating hospital has approved the MIG registry, including the use of "optout" consent. In this model consent is presumed unless the patient "opts out". All participating sites give each patient a "Patient Information Sheet". If a patient informs a staff member that they do not wish to participate, the patient's data are not collected.

Patients who underwent PCI for STEMI and were treated with aspirin and a P2Y 12 inhibitor were included. Exclusion criteria included: thrombolysis prior to PCI; out-of-hospital cardiac arrest; and use of more than one $\mathrm{P}_{2} \mathrm{Y}_{12}$ inhibitor prior to PCI. STEMI was defined as ECG changes (new ST-segment elevation at the J-point or development of Q-waves in two or more contiguous leads) with confirmed myocardial necrosis (elevation in troponin T or I or CK-MB on at least one occasion within 24 hours from index event).

Acute management of all patients including interventional strategy, stent selection and antithrombotic therapy were left to the discretion of the operator in all procedures.

Patients were divided into two groups based on the timing of $\mathrm{P}_{2} \mathrm{Y}_{12}$ inhibitor (clopidogrel, prasugrel or ticagrelor) administration. Those who received a $\mathrm{P}_{2} \mathrm{Y}_{12}$ inhibitor prior to arrival in the catheterisation laboratory were included in the pre-treatment group. Those who received a $\mathrm{P}_{2} \mathrm{Y}_{12}$ inhibitor during or after the PCI were included in the no pre-treatment group.

The primary efficacy endpoint was the proportion of patients who had a TIMI flow $<3$ in the infarcted artery at initial angiography. The primary safety end-point was in-hospital bleeding. 
Secondary outcomes were 12-month mortality and major adverse cardiovascular events (MACE). MACE was defined as the combination of mortality, myocardial infarction (MI), and target vessel revascularization. MI was defined as: an increase in creatine kinase or creatine kinase-MB $\geq 3$ times the upper limit of normal; and/or a significant ST-segment change, development of new $Q$ waves in $\geq 2$ contiguous electrocardiographic leads, or new left branch bundle block pattern in the context of new clinical symptoms. In-hospital bleeding was defined as any bleeding requiring a blood transfusion and/or prolonged hospital admission and/or a drop of haemoglobin $>3 \mathrm{~g} / \mathrm{dL}$.

Continuous variables are expressed as mean $\pm \mathrm{SD}$, and categorical data are expressed as numbers/percentages. Continuous variables were compared using Student's t test. Categorical variables were compared using Fisher's exact or Pearson's chi-square tests as appropriate. Variables were tested for linear trends across the years 2005-2014 using Stata's nptrend command. This is a nonparametric test for trend across ordered groups which is an extension of the Wilcoxon rank-sum test. Multivariate logistic regression was used to estimate the adjusted odds ratio and 95\% confidence interval (CI) for 12-month mortality. Univariate variables with $\mathrm{p}<0.10$ were included in multivariate models. The variables used were: pretreatment, age, DTBT, sex, eGFR, hypertension, diabetes, hypercholesterolaemia, family history of coronary disease, previous MI, previous $\underline{\mathrm{PCI}}$, previous CABG, heart failure, peripheral vascular disease, cerebrovascular disease, left ventricular ejection fraction, multi-vessel CAD, chronic lung disease, cardiogenic shock, glycoprotein IIb/IIIa use, drug-eluting stent use, long stent $(>20 \mathrm{~mm})$, small diameter $(<2.5 \mathrm{~mm})$ and treated lesion location (ostial, bifurcation, left main, LAD, circumflex, right coronary artery, bypass graft).

All statistical analyses were performed using Stata 13.1, StataCorp LP, College Station, TX, USA. P-values $<0.05$ were considered to be statistically significant.

\section{RESULTS}

Of the 3,238 consecutive STEMI patients in the MIG registry from 20052014, 2,807 met the inclusion criteria and were included in this study. Pretreatment with a P2Y12 inhibitor, as well as aspirin, occurred in 892 (31.8\%) 
patients while 1,915 (68.2\%) patients did not receive $\mathrm{P}_{2} \mathrm{Y}_{12}$ inhibitor pretreatment. There was no significant trend in patients receiving pretreatment between 2005 and 2014 despite the availability of prasugrel from 2009 and ticagrelor from 2012 (p-value for trend 0.31). The predominant $\mathrm{P}_{2} \mathrm{Y}_{12}$ inhibitor used was clopidogrel; it was used in $86 \%$ and $77 \%$ of cases in the pretreatment and no pretreatment cohorts.

Baseline clinical characteristics were similar between the two cohorts (Table 1). However, patients in the pretreatment group were more likely to have a history of previous PCI (13.5\% vs. 10.1\%, p<0.01) but had lower rates of Killip class $\geq 2(12.2 \%$ vs. $15.5 \%, \mathrm{p}=0.02)$ and cardiogenic shock (4.5\% vs. $6.7 \%$, $\mathrm{p}=0.02)$ at presentation. They also had longer median door-to-balloon times (75 vs. 70 minutes, $\mathrm{p}=0.03$ ) and overall ischaemic times (197 vs. 186 minutes, $\mathrm{p}=0.01)$.

Procedural characteristics (Table 2) reveal patients in the pretreatment arm were more likely to undergo transradial PCI (13.8\% vs. $10.4 \%, p=0=0.01)$ but less likely to receive a glycoprotein IIb/IIIa inhibitor (69.6\% vs. 76.2\%, $\mathrm{p}<0.01)$ or be treated with a thrombus aspiration catheter $(18.3 \%$ vs. $24.9 \%$, $\mathrm{p}<0.01)$.

There was no difference in the in-hospital primary endpoint of the proportion of patients with TIMI $<3$ flow at initial angiography $(78.0 \%$ vs. $80.7 \%$, $\mathrm{p}=0.18$; Table 3 ) or the safety endpoint of bleeding (3.6\% vs. 3.9\%, p=0.67). Patients in the pretreatment cohort were less likely to suffer in-hospital complications such as cardiogenic shock, new congestive heart failure and arrhythmias. They also had lower in-hospital mortality $(2.3 \%$ vs. $4.4 \%, \mathrm{p}<0.01)$.

Thirty-day and 12-month outcomes are also shown in Table 3. The mortality benefit seen in the pretreatment group during the index admission extended to lower 30 -day (2.8\% vs.5.0\%, p<0.01) and 12-month mortality (4.7\% vs. $7.0 \%, p=0.02$ ). There was no difference between the groups in MACE at 30days or 12-months.

Univariate analysis showed an association between pretreatment and 12month mortality but not for initial TIMI flow, in-hospital bleeding or 12-month MACE. Multivariate logistic regression revealed pretreatment with a $\mathrm{P}_{2} \mathrm{Y}_{12}$ 
inhibitor was not an independent predictor of 12-month mortality (OR 0.79; 95\% CI 0.49-1.28, p=0.32; Figure 1).

\section{DISCUSSION}

Our study shows there has not been a significant trend for change in pretreatment with dual antiplatelet therapy in STEMI over a 10-year period (2005-2014); the overall pretreatment rate was approximately 32\%. An association between pretreatment and lower rates of glycoprotein IIb/IIIa inhibitors and thromboaspiration catheter use was observed. P2Y 12 inhibitor pretreatment was safe without a significant increase in major bleeding but was not associated with improved initial TIMI flow on initial angiography. Although, the pretreatment cohort experienced lower short- and medium-term mortality it was not an independent predictor of 12-month mortality on multivariate analysis, likely due to differences in baseline characteristics between pretreatment versus no pre-treatment groups.

Effective platelet inhibition is essential in the acute management of STEMI with primary PCI.3,4 This concept was validated with the use of glycoprotein IIb/IIIa inhibitors where upstream abciximab infusion was associated with improved coronary artery patency on initial angiography, greater success rate of PCI and improved clinical outcomes. ${ }^{10}$ Thus it is intuitive to assume that administration of aspirin and a $\mathrm{P}_{2} \mathrm{Y}_{12}$ inhibitor as early as possible would translate to improved clinical outcomes. Guidelines uniformly recommend pretreatment however, as evident in our study, it is not routine practice. Our $32 \%$ pretreatment rate is consistent with clinical practice in the United States. ${ }^{11}$ Even with availability and increased use of newer, more potent anti-platelet agents, there has not been a trend towards increase pretreatment either in Australia or the United States. ${ }^{11,12}$ This is likely due to the lack of convincing clinical evidence supporting a pretreatment strategy in patients with STEMI.

A meta-analysis showed a reduction in major adverse cardiovascular events with clopidogrel pretreatment in patients presenting with STEMI. ${ }^{13}$ In our study, the majority of patients in the pretreatment cohort received clopidogrel. Our pretreatment cohort did experience improved survival in-hospital, at 30 days and at 12 months, however pretreatment was not an independent predictor 
of lower mortality. This is most likely explained by the lower risk profile of patients in the pretreatment group who had lower rates of cardiogenic shock and Killip class $\geq 2$ at presentation, and experienced lower rates of new heart failure, cardiogenic shock and significant arrhythmias post PCI. Indeed in our multivariate analysis, cardiogenic shock and left ventricular dysfunction were independent predictors of 12-month mortality. Furthermore, It it is plausible patients with higher risk features did not receive pretreatment due to perceived higher possibility of requiring urgent bypass surgery.

Our results showed pretreatment with a $\mathrm{P}_{2} \mathrm{Y}_{12}$ inhibitor was not associated with an increase in the proportion of patients with TIMI 3 flow at initial angiography. This is consistent with the results from the ATLANTIC trial where pre-hospital administration of ticagrelor did not translate to increased STsegment resolution or improved initial TIMI flow. ${ }^{5}$ The absence of clinical benefit with pre-hospital use of a more potent $\mathrm{P}_{2} \mathrm{Y}_{12}$ inhibitor raises questions about the overall value of a pretreatment strategy. In the context of STEMI, patients with prasugrel or ticagrelor loading exhibit a delay in the onset of anti-platelet effect with a majority having high on-treatment platelet reactivity at one and two hours post loading dose. ${ }^{14} \mathrm{~A}$ potential solution is administration of crushed ticagrelor or prasugrel tablets. This strategy leads to greater platelet inhibition in STEMI when compared to ingestion of integral tablets. ${ }^{15,16}$ As patients who are undergoing primary PCI do not routinely receive crushed $\mathrm{P}_{2} \mathrm{Y}_{12}$ inhibitors, they are unlikely to have adequate platelet inhibition. This may explain the lack of benefit seen with oral $\mathrm{P}_{2} \mathrm{Y}_{12}$ pretreatment.

As there is an apparent lack of benefit from pretreatment, safety becomes an important issue. In our study there was no difference in major bleeding between the two groups. This would be expected as all patients in our study underwent primary PCI and received dual antiplatelet therapy; the only difference being the timing of the loading dose. The safety concern with pretreatment is not in patients who ultimately undergo PCI but in those who require coronary artery bypass surgery or who have non-coronary causes of STelevation. Although rare, in some of these circumstances dual antiplatelet therapy could be potentially catastrophic. ${ }^{17}$ 
Contemporary data indicates that $1-1.6 \%$ of STEMI patients require bypass surgery and over $10 \%$ don't require any revascularization. ${ }^{5}$ Thus these patients are unnecessarily placed at increased risk of bleeding. The unacceptable hazard associated with pretreatment prior to assessing suitability for PCI was shown in the ACCOAST trial. Prasugrel pretreatment in patients with non-ST elevation myocardial infarction (NSTEMI) led to significantly higher bleeding rates. ${ }^{18}$ In contrast, ticagrelor pretreatment in the ATLANTIC trial was not associated with a bleeding hazard in STEMI, irrespective of the definition used. ${ }^{5}$ The difference in population and the larger proportion of patients with STEMI who proceed to PCI may explain the higher risk of bleeding seen with pretreatment in NSTEMI.

We observed that patients in the pretreatment group were less likely to receive a glycoprotein IIb/IIIa inhibitor. It is conceivable the interventionalist may believe that $\mathrm{P}_{2} \mathrm{Y}_{12}$ pretreatment would provide adequate platelet inhibition but this logic is flawed. ${ }^{14}$ Glycoprotein IIb/IIIa inhibitors do provide rapid platelet inhibition but in contemporary practice they were not associated with a reduction in infarct size. ${ }^{19}$ Currently, they are most commonly used in "bail-out" situations such as slow or no-reflow, massive thrombus and/or thrombotic complications. $^{3}$

Cangrelor, a potent intravenous $\mathrm{P}_{2} \mathrm{Y}_{12}$ antagonist with rapid onset and offset, may hold the key in ensuring full platelet inhibition at the time of primary PCI. ${ }^{20}$ Given its rapid onset of action, the infusion can be started once coronary anatomy has been defined thus making pretreatment with oral $\mathrm{P}_{2} \mathrm{Y}_{12}$ agents unnecessary. Cangrelor has been shown to decrease the rate of ischaemic events, including stent thrombosis, across a broad spectrum of PCI patients including those with STEMI. ${ }^{20}$ Although mechanistically attractive, the use of cangrelor has not been examined in STEMI against ticagrelor or prasugrel thus it is uncertain whether it would translate to improved clinical outcomes.

There are some limitations to our study that warrant attention. Firstly, we do not record the exact time of $\mathrm{P}_{2} \mathrm{Y}_{12}$ administration, however drug ingestion occurred in-hospital as ambulance initiated dual antiplatelet therapy is not routine care in our setting. Secondly, although there was a significant increase in the use of prasugrel and ticagrelor since these drugs became available in 2010 
and 2012 respectively, the majority of our patients over the 10 -year period were treated with clopidogrel. ${ }^{12}$ Furthermore, we did not capture whether the clopidogrel patients received a $300 \mathrm{mg}$ or $600 \mathrm{mg}$ loading dose. Thirdly, we did not include patients with ST-elevation who proceeded to urgent angiography but were not treated with PCI. This limits the generalizability of our findings. Finally, given the observational nature of our study there may be other factors, either unmeasured or unaccounted for, that influenced whether patients received a pretreatment strategythe outcome. For example, we do not measure morphine use although it is known to delay gastric emptying and may be associated with worse outcomes in patients with STEMI receiving ticagrelor pretreatment. ${ }^{5}$ CONCLUSION

Pretreatment with a $\mathrm{P}_{2} \mathrm{Y}_{12}$ inhibitor in patients with STEMI is not routine practice in our Australian cohort. Moreover, this strategy is not associated with improved initial TIMI flow at time of angiography or clinical outcomes when

\footnotetext{
baseline clinical and procedural variables are taken into account. Our findings do not provide adequate justification for a strategy of pretreatment. Larger studies are required to definitively ascertain the risk/benefit ratio of dual antiplatelet therapy pretreatment in STEMI

Visualisation of coronary anatomy prior to $\mathrm{P} 2 \mathrm{Y}_{12}$ inhibitor administration would avoid patient exposure to potent antiplatelet agents. This would be advantageous in patients who need urgent coronary artery bypass surgery or those with non-coronary causes of ST-segment elevation.
}

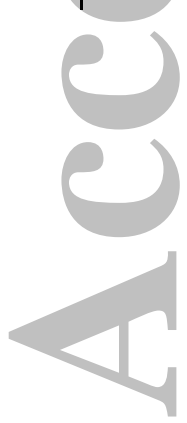


Figure 1. Multivariate Logistic Regression for 12-Month Mortality
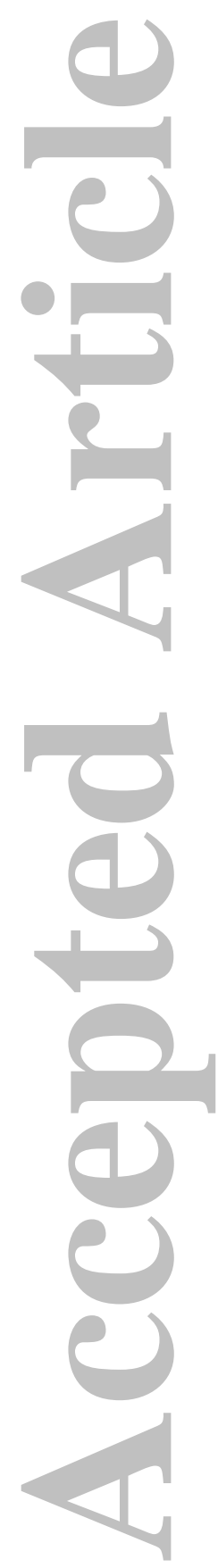


\section{Melbourne Interventional Group Investigators}

MIG Data Management Centre, CCRE, Monash University:

Professor Chris Reid, Dr Nick Andrianopoulos, Ms Angela Brennan, Ms Vibhasha Chand, Dr Diem Dinh.

\section{MIG Steering Committee:}

Professor Chris Reid, A/Professor Andrew Ajani, Dr Stephen Duffy, Dr David Clark, Professor Gishel New, Dr Melanie Freeman, Dr Chin Hiew, Dr Nick Andrianopoulos, Dr Ernesto Oqueli, Ms Angela Brennan.

The following Investigators, Data Managers and Institutions participated in the MIG Database:

Alfred Hospital: SJ Duffy, JA Shaw, A Walton, A Dart, A Broughton, J Federman, C Keighley, C Hengel, KH Peter, D Stub, W Chan, J O’Brien, L Selkrig, K Rankin, R Vandernet, R Huntington, S Pally;

Austin Hospital: DJ Clark, O Farouque, M Horrigan, J Johns, L Oliver, J Brennan, R Chan, G Proimos, T Dortimer, B Chan, V Nadurata, R Huq, D Fernando, A Al-Fiadh, M Yudi, H Sugumar, J Ramchand, H Han. S Picardo, L Brown;

Ballarat Base Hospital: E Oqueli, C Hengel, A Sharma, B Zhu, N Ryan, T Harrison;

Box Hill Hospital: G New, L Roberts, M Freeman, M Rowe, G Proimos, Y Cheong, C Goods, D Fernando, A Teh, S Parfrey, J Ramzy, A Koshy, P Venkataraman, D Flannery;

Geelong Hospital: C Hiew, M Sebastian, T Yip, Michael Mok, C Jaworski, A Hutchinson, C Cimenkaya, P Ngu, B Khialani, H Salehi, M Turner, J Dyson, B McDonald, D Van Den Nouwelant, K Halliburton;

Monash University: H Krum, C Reid, N Andrianopoulos, AL Brennan, V Chand, D Dinh, BP Yan;

Royal Melbourne Hospital: AE Ajani, R Warren, D Eccleston, J Lefkovits, R Iyer, R Gurvitch, W Wilson, M Brooks, S Biswas, J Yeoh.

\section{Acknowledgements:}

The Melbourne Interventional Group acknowledges unrestricted educational grant funding from: Abbott Vascular, Astra-Zeneca, Medtronic, MSD, Pfizer, Servier, and The Medicines Company. These companies do not have access to the data, and do not have the right to review manuscripts before publication.

Dr. Yudi is supported by a combined National Health and Medical Research Council Postgraduate Scholarship (APP 1115163) and a National Heart Foundation Health Professional Scholarship (Award ID 101130). Dr. Duffy's \& Professor Reid's work is funded by National Health and Medical Research Council of Australia Grants.

The Melbourne Interventional Group acknowledges and thanks all patients who have been involved in the registry. 
Conflicts of Interests: The authors report no relationships that could be construed as a conflict of interest.

\section{REFERENCES}

1. Wiviott SD, Braunwald E, McCabe CH, Montalescot G, Ruzyllo W, Gottlieb S, Neumann FJ, Ardissino D, De Servi S, Murphy SA, Riesmeyer J, Weerakkody G, Gibson CM, Antman EM, Investigators T-T. Prasugrel versus clopidogrel in patients with acute coronary syndromes. The New England journal of medicine 2007;357:2001-2015.

2. Wallentin L, Becker RC, Budaj A, Cannon CP, Emanuelsson H, Held C, Horrow J, Husted S, James S, Katus H, Mahaffey KW, Scirica BM, Skene A, Steg PG, Storey RF, Harrington RA, Investigators P, Freij A, Thorsen M. Ticagrelor versus clopidogrel in patients with acute coronary syndromes. The New England journal of medicine 2009;361:1045-1057.

3. Steg PG, James SK, Atar D, Badano LP, Blomstrom-Lundqvist C, Borger MA, Di Mario C, Dickstein K, Ducrocq G, Fernandez-Aviles F, Gershlick AH, Giannuzzi P, Halvorsen S, Huber K, Juni P, Kastrati A, Knuuti J, Lenzen MJ, Mahaffey KW, Valgimigli M, van 't Hof A, Widimsky P, Zahger D. ESC Guidelines for the management of acute myocardial infarction in patients presenting with STsegment elevation. European heart journal 2012;33:2569-2619.

4. O'Gara PT, Kushner FG, Ascheim DD, Casey DE, Jr., Chung MK, de Lemos JA, Ettinger SM, Fang JC, Fesmire FM, Franklin BA, Granger CB, Krumholz HM, Linderbaum JA, Morrow DA, Newby LK, Ornato JP, Ou N, Radford MJ, TamisHolland JE, Tommaso JE, Tracy CM, Woo YJ, Zhao DX, Force CAT. 2013 ACCF/AHA guideline for the management of ST-elevation myocardial infarction: executive summary: a report of the American College of Cardiology Foundation/American Heart Association Task Force on Practice Guidelines. Circulation 2013;127:529-555.

5. Montalescot G, van 't Hof AW, Lapostolle F, Silvain J, Lassen JF, Bolognese L, Cantor WJ, Cequier A, Chettibi M, Goodman SG, Hammett CJ, Huber K, Janzon M, Merkely B, Storey RF, Zeymer U, Stibbe O, Ecollan P, Heutz WM, Swahn E, Collet JP, Willems FF, Baradat C, Licour M, Tsatsaris A, Vicaut E, Hamm CW, Investigators A. Prehospital ticagrelor in ST-segment elevation myocardial infarction. The New England journal of medicine 2014;371:1016-1027.

6. Ajani AE, Szto G, Duffy SJ, Eccleston D, Clark DJ, Lefkovits J, Chew DP, Warren R, Black A, New G, Walton A, Lew R, Shaw J, Horrigan M, Sebastian M, Yan BP, Brennan A, Meehan A, Reid C, Krum H, Melbourne Interventional Group i. The foundation and launch of the Melbourne Interventional Group: a collaborative interventional cardiology project. Heart, lung \& circulation 2006;15:44-47. 7. Chan W, Clark DJ, Ajani AE, Yap CH, Andrianopoulos N, Brennan AL, Dinh DT, Shardey GC, Smith JA, Reid CM, Duffy SJ. Progress towards a National Cardiac Procedure Database--development of the Australasian Society of Cardiac and Thoracic Surgeons (ASCTS) and Melbourne Interventional Group (MIG) registries. Heart, lung \& circulation 2011;20:10-18.

8. Andrianopoulos N, Dinh D, Duffy SJ, Clark DJ, Brennan AL, Chan W, Shardey GC, Smith JA, Yap CH, Buxton BF, Ajani AE, Reid CM. Quality control activities associated with registries in interventional cardiology and surgery. Heart, lung \& circulation 2011;20:180-186. 
9. Lagerqvist B, James SK, Stenestrand U, Lindback J, Nilsson T, Wallentin L, Group SS. Long-term outcomes with drug-eluting stents versus bare-metal stents in Sweden. The New England journal of medicine 2007;356:1009-1019.

10. Montalescot G, Barragan P, Wittenberg O, Ecollan P, Elhadad S, Villain P, Boulenc JM, Morice MC, Maillard L, Pansieri M, Choussat R, Pinton P, Angioplasty AIAbD, Stenting in Myocardial Infarction Regarding A, Long-Term F-u. Platelet glycoprotein IIb/IIIa inhibition with coronary stenting for acute myocardial infarction. The New England journal of medicine 2001;344:1895-1903.

11. Fan $W$, Plent $S$, Prats J, Deliargyris EN. Trends in P2Y12 Inhibitor Use in Patients Referred for Invasive Evaluation of Coronary Artery Disease in Contemporary US Practice. The American journal of cardiology 2016;117:14391443.

12. Yudi MB, Clark DJ, Farouque O, Eccleston D, Andrianopoulos N, Duffy SJ, Brennan A, Lefkovits J, Ramchand J, Yip T, Oqueli E, Reid CM, Ajani AE, Melbourne Interventional G. Clopidogrel, prasugrel or ticagrelor in patients with acute coronary syndromes undergoing percutaneous coronary intervention. Internal medicine journal 2016;46:559-565.

13. Bellemain-Appaix A, O'Connor SA, Silvain J, Cucherat M, Beygui F, Barthelemy O, Collet JP, Jacq L, Bernasconi F, Montalescot G, Group A. Association of clopidogrel pretreatment with mortality, cardiovascular events, and major bleeding among patients undergoing percutaneous coronary intervention: a systematic review and meta-analysis. Jama 2012;308:2507-2516.

14. Alexopoulos D, Xanthopoulou I, Gkizas V, Kassimis G, Theodoropoulos KC, Makris G, Koutsogiannis N, Damelou A, Tsigkas G, Davlouros P, Hahalis G. Randomized assessment of ticagrelor versus prasugrel antiplatelet effects in patients with ST-segment-elevation myocardial infarction. Circulation Cardiovascular interventions 2012;5:797-804.

15. Parodi G, Xanthopoulou I, Bellandi B, Gkizas V, Valenti R, Karanikas S, Migliorini A, Angelidis C, Abbate R, Patsilinakos S, Baldereschi GJ, Marcucci R, Gensini GF, Antoniucci D, Alexopoulos D. Ticagrelor crushed tablets administration in STEMI patients: the MOJITO study. Journal of the American College of Cardiology 2015;65:511-512.

16. Rollini F, Franchi F, Hu J, Kureti M, Aggarwal N, Durairaj A, Park Y, Seawell M, Cox-Alomar P, Zenni MM, Guzman LA, Suryadevara S, Antoun P, Bass TA, Angiolillo DJ. Crushed Prasugrel Tablets in Patients With STEMI Undergoing Primary Percutaneous Coronary Intervention: The CRUSH Study. Journal of the American College of Cardiology 2016;67:1994-2004.

17. Chatterjee S. ECG Changes in Subarachnoid Haemorrhage: A Synopsis. Neth Heart J 2011;19:31-34.

18. Montalescot $G$, Bolognese $L$, Dudek $D$, Goldstein $P$, Hamm $C$, Tanguay JF, ten Berg JM, Miller DL, Costigan TM, Goedicke J, Silvain J, Angioli P, Legutko J, Niethammer M, Motovska Z, Jakubowski JA, Cayla G, Visconti LO, Vicaut E, Widimsky P, Investigators A. Pretreatment with prasugrel in non-ST-segment elevation acute coronary syndromes. The New England journal of medicine 2013;369:999-1010.

19. Mehilli J, Kastrati A, Schulz S, Frungel S, Nekolla SG, Moshage W, Dotzer F, Huber K, Pache J, Dirschinger J, Seyfarth M, Martinoff S, Schwaiger M, Schomig A, Bavarian Reperfusion Alternatives Evaluation-3 Study I. Abciximab in patients with acute ST-segment-elevation myocardial infarction undergoing primary 
percutaneous coronary intervention after clopidogrel loading: a randomized double-blind trial. Circulation 2009;119:1933-1940.

20. Bhatt DL, Stone GW, Mahaffey KW, Gibson CM, Steg PG, Hamm CW, Price MJ, Leonardi S, Gallup D, Bramucci E, Radke PW, Widimsky P, Tousek F, Tauth J, Spriggs D, McLaurin BT, Angiolillo DJ, Genereux P, Liu T, Prats J, Todd M, Skerjanec S, White HD, Harrington RA, Investigators CP. Effect of platelet inhibition with cangrelor during PCI on ischemic events. The New England journal of medicine 2013;368:1303-1313.
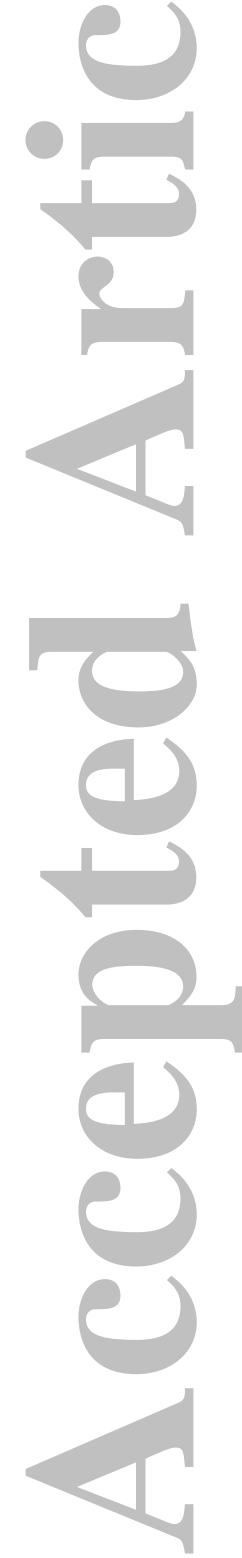


\section{University Library}

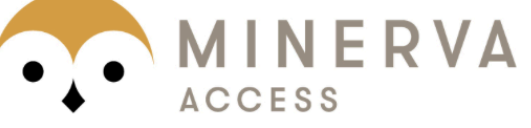

A gateway to Melbourne's research publications

Minerva Access is the Institutional Repository of The University of Melbourne

\section{Author/s:}

Yudi, MB;Farouque, O;Andrianopoulos, N;Ajani, AE;Brennan, A;Lefkovits, J;Reid, CM;Chan, W;Duffy, SJ;Clark, DJ

Title:

Pretreatment with dual antiplatelet therapy in patients with ST-elevation myocardial infarction

Date:

2018-08-01

\section{Citation:}

Yudi, M. B., Farouque, O., Andrianopoulos, N., Ajani, A. E., Brennan, A., Lefkovits, J., Reid, C. M., Chan, W., Duffy, S. J. \& Clark, D. J. (2018). Pretreatment with dual antiplatelet therapy in patients with ST-elevation myocardial infarction. CATHETERIZATION AND CARDIOVASCULAR INTERVENTIONS, 92 (2), pp.E98-E105. https://doi.org/10.1002/ ccd.27325.

Persistent Link:

http://hdl.handle.net/11343/293520 